\title{
Electronic Structure of Dangling and Floating Bonds in Amorphous Silicon
}

\author{
R. Biswas, ${ }^{(1)}$ C. Z. Wang, ${ }^{(1)}$ C. T. Chan, ${ }^{(2)}$ K. M. Ho, ${ }^{(2)}$ and C. M. Soukoulis ${ }^{(2)}$ \\ (1)Department of Physics and Microelectronics Research Center, Iowa State University, Ames, Iowa 50011 \\ (2) Ames Laboratory-U.S. DOE, Iowa State University, Ames, Iowa 50011 \\ (Received 19 July 1989)
}

\begin{abstract}
The electronic structure of amorphous silicon models has been calculated with the tight-binding parameters of Chadi. An energy gap of $\sim 1.0 \mathrm{eV}$ is obtained for a fully four-coordinated model, whereas gap states occur for models with coordination defects. Dangling-bond gap states are well localized on the three-coordinated site. Floating bonds lead to considerably less localized gap states with wavefunction amplitudes on the neighbors of the five-coordinated atom. The hyperfine splittings of these coordination defects are calculated and compared to experiment.
\end{abstract}

PACS numbers: $71.25 . \mathrm{Mg}, 61.40 .+\mathrm{b}$

The dominant paramagnetic defect in amorphous silicon $(a-\mathrm{Si})$ has been commonly believed to be the dangling bond. Recently, however, Pantelides proposed that the floating bond or five-coordinated silicon atom is an equally viable native defect in $a-\mathrm{Si}^{1,2}$

However, Phillips ${ }^{3}$ has argued that the growth kinetics of $a$-Si films is unfavorable for overcoordination defects, but should instead favor dangling bonds as the dominant defect. Stutzmann and Biegelsen ${ }^{4}$ observed that the ${ }^{29} \mathrm{Si}$ hyperfine data are completely consistent with the dangling-bond model. Based on defect-state wave functions and energies for Bethe-lattice-terminated nineatom Si clusters, Fedders and Carlsson ${ }^{5,6}$ inferred that the ESR signal in $a-\mathrm{Si}: \mathrm{H}$ arises from dangling bonds.

In this paper, we wish to clarify the controversy between the dangling and floating bonds by calculating their electronic properties and presenting new results on the differences between these two types of coordination defects that should be useful for the interpretation of experimental data. Structural models containing both dangling and floating bonds have been derived by Biswas, Grest, and Soukoulis ${ }^{7}$ (BGS) from molecular-dynamics simulations of the quenching of a melt of bulk $\mathrm{Si}$. In contrast to previous calculations, ${ }^{5,6}$ we do not ascribe a specific structural geometry to the floating bond, but instead allow it to assume an energetically favorable configuration consistent with the amorphous network topology. To meaningfully extract properties of the coordination defects, we have also performed calculations for the Monte Carlo $a-\mathrm{Si}$ model generated by Wooten, Winer, and Weaire ${ }^{8,9}$ (W3), in which all atoms have a coordination of four.

The electronic-structure calculations were performed with a nearest-neighbor tight-binding model consisting of the minimal basis of one $s$ and three $p$ Si orbitals and the tight-binding parameters derived by Chadi. ${ }^{10}$ The Chadi tight-binding model has been extensively used to study structural, electronic, and vibrational properties of crystalline $\mathrm{Si}$ and $\mathrm{Si}(111)$ and $\mathrm{Si}(100)$ surfaces with much success. We have incorporated the standard $1 / d^{2}$ scaling of the interatomic matrix elements, together with a bond cutoff that is between the first and second peaks of the radial distribution function $g(r)$ of the $a$-Si models. The interatomic interaction is described by the sum of the electronic band-structure energy, and a repulsive twobody potential is derived ${ }^{11}$ by subtracting the bandstructure energy from accurate first-principles totalenergy calculations for the diamond Si structure. ${ }^{12}$

The atomic positions obtained by the classical W3 and BGS models (216 atoms) were used as input for the tight-binding electronic-structure approach and the positions were then relaxed to the lowest energy. Within the Chadi parametrization, a well-defined gap of $-1.0 \mathrm{eV}$ exists for the four-coordinated relaxed W3 model (see Fig. 1). The valence density of states (DOS), especially the position of the $p$ peak and the bandwidth, is in very good agreement with photoemission measurements. ${ }^{13}$ The conduction band, however, is not given accurately from Chadi's scheme, but this is less important for our studies. In contrast to the $\mathrm{W} 3$ case, the relaxation of the BGS atomic positions substantially reduced the gap DOS from the structure obtained with the unrelaxed po-

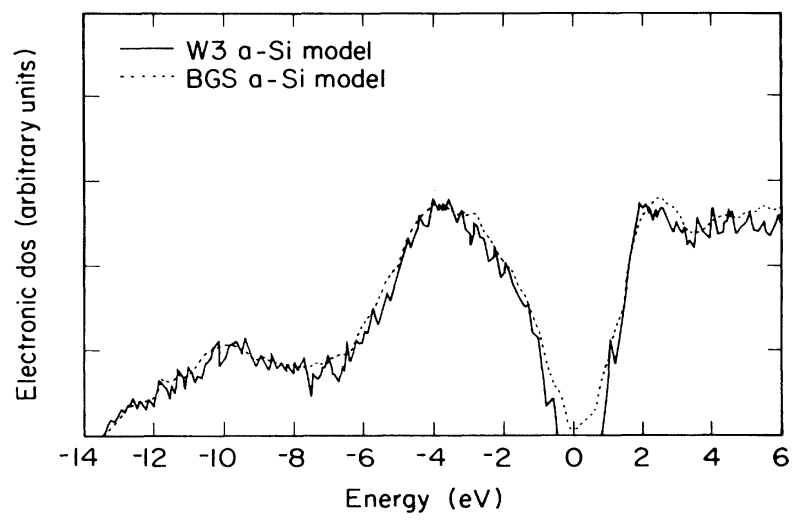

FIG. 1. Electronic densities of states for the W3 model (solid line) compared to the BGS model (broken line). All sites of the W3 model have coordination four, whereas the BGS model has three- and five-coordinated atoms. 


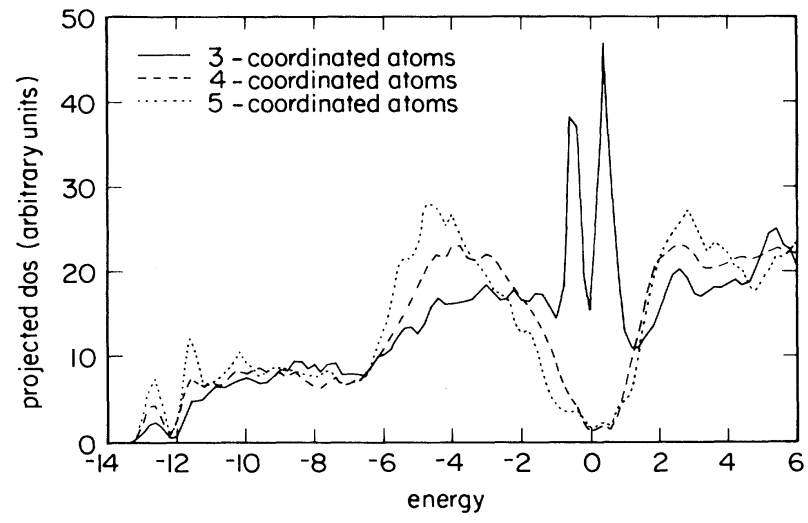

FIG. 2. Projected electronic densities of states on three-, four-, and five-coordinated atoms in the BGS model.

sitions. ${ }^{7}$ After this relaxation step, the BGS model had twelve dangling bonds and four floating bonds.

We find that the DOS in the gap region arises from either (i) dangling bonds, (ii) floating bonds, or (iii) strongly disordered four-coordinated sites. The projected DOS on sites of different coordination (Fig. 2) displays the large contributions of dangling bonds to the gap states. Within the Chadi model, neutral $\left(D^{0}\right)$, negatively charged $\left(D^{-}\right)$, and positively charged $\left(D^{+}\right)$ dangling-bond states exist. The relative positions of these charge states cannot be determined reliably with the present scheme, although the $D^{0}, D^{-}$, and $\left(D^{+}\right)$levels comprise the lower (upper) peaks. Since the Chadi parameters can overemphasize charge-transfer effects, a Hubbard on-site repulsion $(U)$ of $1.0 \mathrm{eV}$ was used in the calculations of Fig. 2, similar to previous calculations for the $\mathrm{Si}(111)$ surface. ${ }^{14}$

The wave functions of the dangling bonds are very strongly localized. We found two strongly localized midgap states in each of which $\sim 57 \%$ of the wave function was centered on the dangling bond, together with very small amplitudes on neighboring atoms [Fig. 3(a)]. The finite size of our $a-\mathrm{Si}$ model resulted in the hybridization among dangling-bond states. Interactions between defects in finite-size models have been noted in previous work. ${ }^{15}$ To isolate individual dangling bonds, we introduced "terminator" atoms on all dangling bonds except the one being studied. The terminators had a single $s$ orbital with a site energy $E_{s}=0$ and the same $s s$ and $s p$ interactions as in Si. The terminators played a role similar to $\mathrm{H}$ in $a-\mathrm{Si}: \mathrm{H}$ in reducing gap states and opening a gap of $0.45 \mathrm{eV}$ in our model. This led to gap states in which $40 \%-50 \%$ of the wave function was localized on individual dangling bonds. These strongly localized dangling-bond states are consistent with ESR results.

Saturating all dangling bonds in our model with termi-

nator atoms removes the interactions between dangling and floating bonds among the gap states. Each of the four floating bonds in the model lead to a separate state either in the valence-band tail or in the gap region. In each of these states (Table I), the amplitude of the wave function is vanishingly small on the central five-coordinated atom, but is dis- (a)

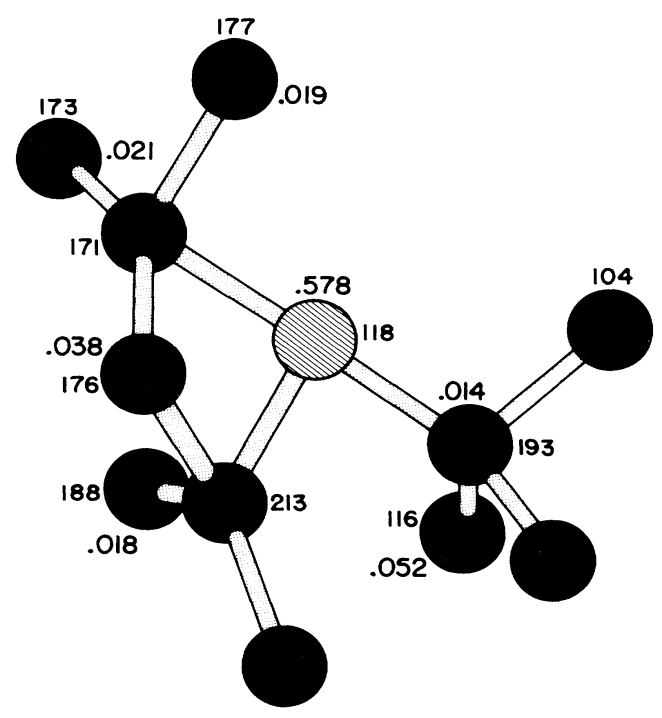

(b)

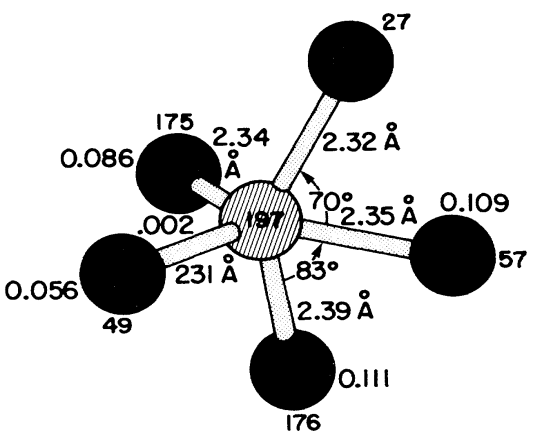

FIG. 3. Local structural geometries at (a) a dangling-bond site, (b) a floating-bond site, and (c) a highly distorted fourcoordinated atom. The amplitudes of the wave functions of the gap states generated by each configuration are shown, together with representative bond lengths and bond angles.

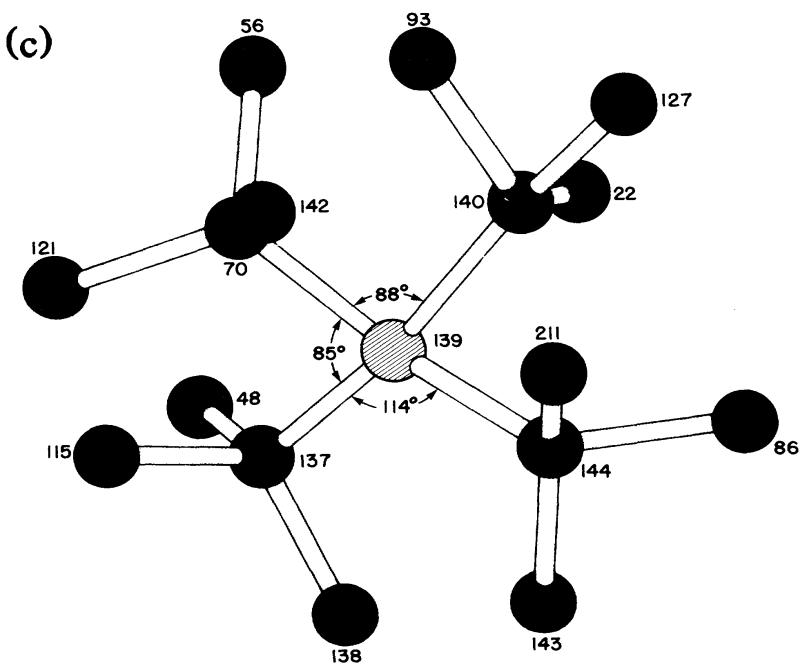


TABLE I. Wave-function amplitudes, $s$ and $p$ components, and hyperfine shifts in the immediate environment of the floating bonds ( $\mathrm{fb}$ ) and dangling bonds (db).

\begin{tabular}{|c|c|c|c|c|c|c|c|}
\hline $\begin{array}{c}\text { Energy } \\
(\mathrm{eV})\end{array}$ & Atom & Coordination & $\eta_{j}^{2}$ & $\alpha_{j}^{2}$ & $\beta_{j}^{2}$ & $a_{u}^{2} \eta_{j}^{2}$ & $\begin{array}{l}\Delta H \\
(G) \\
\end{array}$ \\
\hline-0.48 & 214 & 5 & 0.003 & & & & \\
\hline \multirow[t]{5}{*}{$\mathrm{fb}$} & 49 & 4 & 0.147 & 0.31 & 0.69 & 0.046 & 67 \\
\hline & 192 & 4 & 0.131 & 0.22 & 0.78 & 0.029 & 42 \\
\hline & 43 & 4 & 0.104 & 0.23 & 0.77 & 0.024 & 35 \\
\hline & 67 & 4 & 0.036 & 0.08 & 0.92 & 0.003 & 4 \\
\hline & 194 & 4 & 0.023 & 0.16 & 0.84 & 0.004 & 5 \\
\hline 0.25 & 165 & 5 & 0.003 & & & & \\
\hline \multirow[t]{4}{*}{$\mathrm{fb}$} & 211 & 4 & 0.184 & 0.13 & 0.87 & 0.024 & 36 \\
\hline & 199 & 4 & 0.124 & 0.07 & 0.93 & 0.009 & 13 \\
\hline & 93 & 4 & 0.123 & 0.08 & 0.92 & 0.010 & 14 \\
\hline & 145 & 4 & 0.066 & 0.22 & 0.78 & 0.015 & 22 \\
\hline \multicolumn{2}{|c|}{ Si(111) ideal } & 3 & & 0.191 & 0.809 & & \\
\hline \multicolumn{2}{|c|}{$\mathrm{Si}(111) \Delta z=0.53 \AA$} & 3 & & 0.106 & 0.894 & & \\
\hline 0.59 & 118 & 3 & 0.573 & 0.01 & 0.99 & 0.005 & 7 \\
\hline \multirow[t]{3}{*}{$\mathrm{db}$} & 116 & 4 & 0.052 & 0.37 & 0.63 & 0.020 & 29 \\
\hline & 176 & & 0.038 & 0.12 & 0.88 & 0.005 & 7 \\
\hline & 171 & & 0.031 & 0 & 1.0 & 0 & 0 \\
\hline 0.79 & 103 & 3 & 0.401 & 0.04 & 0.96 & 0.016 & 23 \\
\hline \multirow[t]{4}{*}{$\mathrm{db}$} & 176 & 4 & 0.065 & 0.31 & 0.69 & 0.020 & 30 \\
\hline & 172 & 4 & 0.039 & 0.44 & 0.56 & 0.017 & 25 \\
\hline & 173 & 4 & 0.035 & 0.33 & 0.67 & 0.012 & 17 \\
\hline & 171 & 4 & 0.031 & 0.63 & 0.37 & 0.016 & 23 \\
\hline
\end{tabular}

tributed over 3-5 four-coordinated neighbors of the five-coordinated atom. More than half of the wave function is delocalized outside this six-atom cluster. The anisotropic nature of the wave function can be observed in Fig. 3(b). These conclusions are consistent with the results of Fedders and Carlsson ${ }^{5,6}$ for canonical floatingbond configurations.

These results for the floating-bond wave function are physically plausible, since the four $s p^{3}$ hybrids on the five-coordinated atom should be occupied. The extra floating bond then leads to an unpaired electron spin on the neighboring atoms. Our results indicate this unpaired electron is somewhat delocalized and can resonate between three or four of the neighbors. We stress that the picture of the floating bond consisting of a single dominant site involving an unpaired electron spin with one secondary atom ${ }^{16}$ is inconsistent with our results.

A significant difference in the structural geometry at the five-coordinated sites in our $a-\mathrm{Si}$ model and the "ideal" floating-bond configuration is the absence of three collinear atoms. The $180^{\circ}$ bond angle associated with the collinear geometry generates considerable angular strain which is relieved by a lower-symmetry structural distortion.

Electron-spin-resonance spectra ${ }^{17}$ in $a-\mathrm{Si}: \mathrm{H}$ indicate a hyperfine line with a splitting of $\sim 74 \mathrm{G}$ from the central ESR signal, arising from the interaction of an unpaired electronic spin with a ${ }^{29} \mathrm{Si}$ nuclear spin. The hyperfine data have been interpreted as evidence both for ${ }^{16}$ and against ${ }^{18}$ the floating-bond configuration. We examine the hyperfine splitting expected in our model, which is given by

$$
\Delta H=\left(\frac{16 \pi}{3}\right)\left(\frac{\mu_{j}}{I_{j}}\right) \mu_{B} \alpha_{j}^{2} \eta_{j}^{2}\left|\psi_{3 s}(0)\right|^{2} .
$$

Here $\mu_{j}$ and $I_{j}$ are the nuclear magnetic moment and nuclear spin of ${ }^{29} \mathrm{Si} . \mu_{B}$ is the Bohr magneton. $\alpha_{j}, \beta_{j}$, and $\eta_{j}$ are the $s$ and $p$ components and amplitudes of the electronic wave function on site $j$ defined by $|\psi\rangle=\sum_{j} \eta_{j}\left(\alpha_{j}\left|s_{j}\right\rangle+\beta_{j}\left|p_{j}\right\rangle\right)$, with $\alpha_{j}^{2}+\beta_{j}^{2}=1$. They are given in Table $I$. We adopted the value of $31.5 \times 10^{-24}$ $\mathrm{cm}^{-3}$ from Ref. 19 for the electronic valence $s$ wave function at the nuclear site.

The floating-bond wave functions have large $s$ components $\alpha_{j}$. Although the individual amplitudes $\eta_{j}$ on the sites surrounding the five-coordinated atom are small, a substantial product $\alpha_{j}^{2} \eta_{j}^{2}$ is possible, resulting in a distribution of hyperfine splittings between $\sim 25 \mathrm{G}$ and $67 \mathrm{G}$ (Table I). This leads to an overall broadening of the ESR line, rather than a sharp hyperfine feature. The distribution of hyperfine splittings arises from the delocalized nature of the floating-bond wave function.

The $s$ and $p$ characters of the dangling bonds are very sensitive to the structural geometry. In our $a-\mathrm{Si}$ model, the dangling bonds are mostly $p$-like with $\alpha_{j}^{2}$ between 0.001 and 0.05 , which leads to very small hyperfine split- 
tings. Results for the other dangling bonds are very similar to the representative results in Table $I$. This is in direct contrast to the $\mathrm{Si}(111)$ surface dangling bonds, where the $s$ character is much larger, with $\alpha_{j}^{2}$ varying from 0.19 for the unreconstructed case to $\sim 0.10$ for an inward surface relaxation of $0.53 \AA$ (Table I). The surface calculations were also performed with the present tight-binding model which, in fact, energetically favors the unrelaxed $\mathrm{Si}(111)$ configuration rather than a more planar geometry. The reduced $s$ character of the $a$ $\mathrm{Si-model} \mathrm{dangling} \mathrm{bonds} \mathrm{is} \mathrm{due} \mathrm{to} \mathrm{large} \mathrm{bond-angle} \mathrm{dis-}$ tortions at these sites [e.g., Fig. 3(a)]. We emphasize that both strong localization as well as substantial $s$ character is needed to account for the hyperfine spectra. One interpretation is that the observed hyperfine spectra could arise from a class of dangling bonds that have a narrow structural distribution very similar to the Si(111) surface. Such dangling bonds may occur on the surfaces of microvoids.

Finally, strongly disordered four-coordinated atoms in both the BGS and $\mathrm{W} 3$ model give rise to DOS peaks in the valence-band tail. These states emerge in DOS projected on individual atoms and are relatively insensitive to the presence of terminator atoms. Four-member rings of atoms, that have large bond-angle deviations, lead to states in the valence tail (between -0.8 and $-0.6 \mathrm{eV}$ ). Individual atoms with large bond-angle deviations $\Delta \boldsymbol{\theta}_{\mathrm{rms}}$ exceeding $25^{\circ}$ [see Fig. 3 (c)], or weak $\mathrm{Si}-\mathrm{Si}$ bonds, lead to states that are less localized than either the danglingor floating-bond states. The large structural distortions pull out bonding and antibonding states from the valence and conduction bands and bring them towards midgap, resulting in band-tail states.

In conclusion, floating bonds have substantially less localized wave functions than dangling bonds. Their wave functions are centered on the neighbors of the fivecoordinated atom giving rise to a distribution of hyperfine splittings.

We note, however, that there may be kinetic factors that do not favor five-coordinated atoms. In previous work, ${ }^{20}$ we found that introduction of microvoids in $\mathbf{a}-\mathrm{Si}$ networks decreased by an order of magnitude the number of floating bonds. This is due to the ability of the floating bonds to diffuse easily and reach an internal surface, where they could recombine with dangling bonds. Discussion of such kinetic factors is an aspect for further study.

We thank S. Pantelides and J. C. Phillips for many valuable suggestions and comments, and G. S. Grest, J. Shinar, and B. N. Harmon for helpful discussions. R.B. and C.M.S. acknowledge support from the Electric Power Research Institute under Contract No. RP 30701. We acknowledge a National Science Foundation grant of supercomputer time at the National Center for Supercomputing Applications and support from the Air Force Office of Scientific Research. Ames Laboratory is operated by Iowa State University for the U.S. Department of Energy (U.S. DOE) under Contract No. W7405-ENG-82 and is supported by the Director for Energy Research, Division of Material Sciences, U.S. DOE.

'S. T. Pantelides, Phys. Rev. Lett. 57, 2979 (1986); 58, 1344 (1987).

2J. H. Stathis and S. T. Pantelides, Phys. Rev. B 37, 6579 (1988).

${ }^{3}$ J. C. Phillips, Phys. Rev. Lett. 58, 2824 (1987).

${ }^{4}$ M. Stutzmann and D. K. Biegelsen, Phys. Rev. Lett. 60, 1682 (1988).

${ }^{5}$ P. A. Fedders and A. E. Carlsson, Phys. Rev. B 37, 8506 (1988).

6P. A. Fedders and A. E. Carlsson, Phys. Rev. B 39, 1134 (1989).

${ }^{7}$ R. Biswas, G. S. Grest, and C. M. Soukoulis, Phys. Rev. B 36, 7437 (1987).

${ }^{8}$ F. Wooten, K. Winer, and D. Weaire, Phys. Rev. Lett. 54, 1392 (1985).

${ }^{9}$ K. Winer, Phys. Rev. B 35, 2366 (1987).

${ }^{10}$ D. J. Chadi, Phys. Rev. B 19, 2074 (1979).

${ }^{11}$ C. Z. Wang, C. T. Chan, and K. M. Ho, Phys. Rev. B 39, 8586 (1989).

${ }^{12}$ M. T. Yin and M. L. Cohen, Phys. Rev. B 26, 5668 (1982).

${ }^{13}$ L. Ley, in The Physics of Hydrogenated Amorphous Silicon II, edited by J. D. Joannopoulos and G. Lucovsky (Springer-Verlag, Berlin, 1984), p. 61.

${ }^{14}$ O. Alerhand and E.J. Mele, Phys. Rev. Lett. 59, 657 (1987).

${ }^{15}$ L. Martin-Moreno and J. A. Vergés, Phys. Rev. B 39, 3445 (1989).

${ }^{16}$ J. H. Stathis, Phys. Rev. B 40, 1232 (1989).

${ }^{17}$ D. K. Biegelsen and M. Stutzmann, Phys. Rev. B 33, 3006 (1986).

${ }^{18}$ M. Cook and C. T. White, Mater. Res. Soc. Symp. Proc. 141, 85 (1989).

${ }^{19}$ G. W. Watkins and J. W. Corbett, Phys. Rev. 134, A1359 (1964).

${ }^{20}$ R. Biswas, I. Kwon, A. M. Bouchard, C. M. Soukoulis, and G. S. Grest, Phys. Rev. B 39, 5101 (1989). 